\title{
Several erosion test results of means of sand control
}

\author{
Alexey Alkhimenko, Nikita Shaposhnikov, Boris Shemyakinsky, and Anton Tsvetkov* \\ Peter the Great St. Petersburg Polytechnic University, 195251 St. Petersburg, Russia
}

\begin{abstract}
When screens are used as the sole means of sand control, they function as a downhole filter. The entry of sand or other particles into horizontal and vertical wells presents the problem of steel erosion and productivity lost. Downhole is the most desirable location to restrict its access into the well. The problem of material reliability testing is relevant today, that's why several erosion tests were made and studied. Selfmade test bench allow varying velocity flow and fractions conditions (composition, quantity, size, hardness). Slurry erosion experiments resulted in wear of downhole sand control screens. The wear criteria were checked by weight loss of screen samples material and its integrity degradation. The idea of slurry erosion tests was to reveal screens wear.
\end{abstract}

\section{Introduction}

The fuel-power complex holds one of the leading positions in Russian economy. Petroleum production connected with the specific conditions of equipment operation: high temperature and pressure, corrosion and erosion of the downhole material, friction and wear of drill and casing pipes and so on. Erosive wear is one of a major concern in oil industry [1], and downhole screen slurry erosion, in particular, has increased in importance with the development of its complexity and cost. Along with this, slurry erosion is common in oil well fluid handling systems. According to [2] sand is commonly produced along with production oil, and this is also a problem for the industry.

Based on this, steel reliability testing is relevant today not only for estimation of essential safe mode operating conditions, but also because of the need to know under which of them breakages and failures of the equipment, caused by continual use in hard circumstances, will come and interface to economic loss.

Slurry erosion is opposed from classical wear in that there is a fluid contribution to the mechanical action that is producing wear and increase material removal [3]. The entry of sand or other particles into horizontal and vertical wells presents troubles of material erosion and productivity lost.

Stand-alone screen installations function as a downhole filter. But screens prevention of particles (entrained with the produced fluids) less than $50 \mu \mathrm{m}$ is difficult to imagine [2]. Small particles can pass through, but then the bigger ones blocks several slots and cause erosion, because of the increasing velocity in other sections of the screens. Therefore screen slots opens and allow particles with larger diameter passing, eroding and cause failure.

In accordance with [4] erosion rate is mostly governed by mass of particles per unit of fluid, fractions density and diameter, fluid velocity, impingement angle of incidence, screen material properties, its surface application and so on. Downhole sand control screen wear tests in different conditions are rather common for erosion research in petroleum material science and reflected in various surveys such as [5-8]. Thus erosive wear failures of downhole screens are difficult to prevent.

The erosion of materials, caused by impact of hard particles, generally classified as wear. It is a form of material degradation. Referring to [9], erosion is a gradual process of mechanical wear that removes the material by recurrent deformation and cutting actions due to the solid impingement. In oil and gas well completions, screen wear can occur from two main mechanisms: sandblasting erosion and "wormhole" erosion, caused by highly localized fluid velocities passing through the screen. The most efficient energy transfer from the particle to the surface and, at the same time, the most erosion impact of the slurry stream, would appear at angle $90^{\circ}$ to the surface [10]. In our case, test bench's flow loop provides this angle of solids flow rate.

\section{Methodology}

Present erosion tests were made on a special wear testing bench. Test bench was designed for erosion wear test of samples with various geometry (including screens). Test bench's flow loop (fig. 1) provides flow rate of solids up to $45 \mathrm{gpm}$ (max. allowed particles diameter $\sim 700$ $\mu \mathrm{m})$ with pressure - up to 25 bar. Developed software records the following parameters: flow velocity, fluid temperature, system pressure and pump motor power.

Screen samples were made from downhole screen elements. Test was performed for samples named by 10 8 (weight before test - 107,346 gr) and 12-3 (weight before test - 101,631 gr).

$\overline{{ }^{*} \text { Corresponding author: a.s.tsvetkow@gmail.com }}$ 


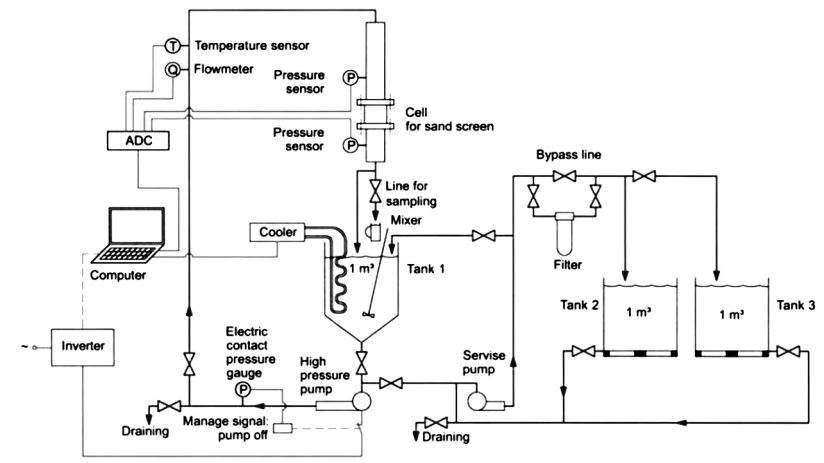

Fig. 1. Test bench's flow loop setup scheme

Each screen sample was impinged with quartz sand or silicon carbide particles introduced into a highvelocity slurry stream. The required flow velocity (45 gpm) was achieved by the flow rate and the insert diameter: insert $-0,4$ in; offset $-0,5$ in.

Distribution of wear allowed allocating special areas of erosion: high eroded diameter (HED), visible wear diameter (VWD), erosion wear diameter (EWD). Test wear areas were determined as: VWD - 2,3 in, EWD 1,3 in, HED - 1,1 in.

Test samples erosive wear evaluation was made by weight loss calculation and slot size degradation measurement. Before weighing samples were dried and cleaned.

\section{Results and discussion}

Erosive wear changes and test results are summarized in table 1.

Table 1. Impingement test results

\begin{tabular}{|c|c|c|c|c|c|c|}
\hline № & $\begin{array}{c}\text { Test, } \\
\text { hr }\end{array}$ & $\begin{array}{l}\text { Part. } \\
\text { conc. }\end{array}$ & $\begin{array}{c}\text { Weight, } \\
\text { gr }\end{array}$ & $\begin{array}{c}\text { VWD } \\
\%\end{array}$ & $\begin{array}{c}\text { EWD } \\
\%\end{array}$ & $\begin{array}{c}\text { HED } \\
\%\end{array}$ \\
\hline \multirow{3}{*}{$10-8$} & 0.25 & \multirow{3}{*}{4500} & 105.065 & 3.83 & 11.59 & 17.70 \\
\hline & 0.75 & & 102.510 & 4.28 & 12.98 & 19.82 \\
\hline & 1.75 & & 101.267 & 2.08 & 6.31 & 9.64 \\
\hline \multirow{4}{*}{$12-3$} & 0.25 & \multirow{4}{*}{1500} & 100.812 & 1.37 & 4.16 & 6.35 \\
\hline & 0.75 & & 99.560 & 2.10 & 6.36 & 9.71 \\
\hline & 1.75 & & 98.457 & 1.85 & 5.61 & 8.56 \\
\hline & 3.75 & & 97.120 & 2.24 & 6.79 & 10.37 \\
\hline
\end{tabular}

Impingement test of 12-3 sample was typified by stages: incubation (from 0,25 to $0,75 \mathrm{hr}$ ); accelerated attack (from 0,75 to $1,75 \mathrm{hr}$ ); steady-state (from 1,75 to $3,75 \mathrm{hr}$ ). The direct impingement erosion tests shown that impingement tests must be stopped on incubation stage.

Sand cycle test conducted to reveal screen sample wear and to keep constant degradation rate of abrasive particles. Therefore it is crucial to determine their wear rate for keeping constant average particle size distribution (PSD) during the experiment.

Decreasing of wear rate during testing can be attributed to the following factor. Due to a significant slot size increase in the zone of direct impact to the sizes enabling passing of all abrasive particles, a new flow with higher velocity is generated that significantly increases local wear and reduces the impact of sliding wear. Wear mainly develops in the zone of direct impact through degraded slots.

\section{Conclusions}

Slurry erosion wear is a serious problem that can bring equipment to failure. The problem of material reliability testing is relevant today, that's why impingement tests were studied. Tests were typified by stages and material removal was monitored with time. Results shows that large particles striking caused more damage than small ones.

The severity of the erosion rate depends on many factors such as velocity rate of particles, their composition, quantity, diameter and hardness. Erosion tests require careful control of its conditions (including PSD). Conveying of solid particles caused screens erosive wear. But abrasive particles are wearing off too simultaneously with screen samples.

Further enhancement to the tools and its applications are on-going. Gained results of the present study will be used further to determine the erosion potential for a given equipment design and operating condition. Next stage is to predict the extent of wear and failure conditions of standalone screens for downhole application to enhance production reliability.

\section{References}

1. J. Zhang, et al. J. Nat. Gas Sci. Eng. 32, 334-346 (2016)

2. M. Parsi, et al. J. Nat. Gas Sci. Eng. 21, 850-873 (2014)

3. K.G. Budinski. Guide to friction, wear, and erosion testing. ASTM International. (2007)

4. C.B. Solnordal, et al. Wear. June, 205-215 (2013)

5. S.A. Ali, et al. Petroleum Eng. Int. 69 (7), 36-41 (1996)

6. G. Colwart, et al. Drilling contractor. May/June, 118-125 (2007)

7. M.S.A. Zamberi, et al. Proceedings of the Annual Offshore Technology Conference. Kuala Lumpur, Malaysia. (2014)

8. A.Yu. Valdberg, et al. Chem. and Pet. Eng. 47, 369$373(2011)$

9. M.A. Islam, et al. Wear. 311 (1-2), 180-190 (2014)

10. A. Levy. Solid Particle Erosion and ErosionCorrosion of Materials. ASTM International. (1995) 